Original

\title{
Comparison of efficacy and outcome satisfaction between in-office and home teeth bleaching in Chinese patients
}

\author{
Jie Nie1), Fu-Cong Tian'1), Zu-Hua Wang1), Adrian U. Yap"2,3,4), and Xiao-Yan Wang1) \\ 1)Department of Cariology and Endodontology, Peking University School and Hospital of Stomatology, \\ Beijing, P. R. China \\ ${ }^{2}$ Department of Dentistry, Ng Teng Fong General Hospital, National University Health System, Singapore \\ 3)School of Science and Technology, Singapore University of Social Sciences, Singapore \\ 4)Faculty of Dentistry, National University of Singapore, Singapore
}

(Received August 30, 2016; Accepted December 17, 2016)

\begin{abstract}
This in vivo study aimed to evaluate the efficacy of in-office and home teeth bleaching in Asian patients. Moreover, the correlation between tooth color change and patient's outcome satisfaction was investigated. Overall, 40 Chinese patients were randomly divided into two groups and prescribed in-office (OB) or take-home bleaching (HB). The color of the maxillary central incisor and canine were recorded at baseline, immediately after first treatment, 1 week, and 3 months after treatment by using a spectrophotometer. Bleaching sensitivity and outcome satisfaction were assessed using Likert and visual analogue scale (VAS), and the results were analyzed using independent $t$-test and Pearson correlation $(P$ $<0.05) . \Delta \mathrm{L}^{*}, \Delta \mathrm{a}^{*}, \Delta \mathrm{b}^{*}$, and $\Delta \mathrm{E}^{*}$ values of $\mathrm{HB}$ were greater than those of $\mathrm{OB}$. Color changes observed in canines were generally greater than those in incisors. Moreover, HB was generally associated with less tooth sensitivity than OB. Patients were satisfied with both treatments, but VAS scores were greater for HB. Furthermore, the correlation between $\Delta \mathrm{E}^{*}$ and VAS was significant for canines immediately after the first treatment and 1 week after HB. In conclusion, HB
\end{abstract}

Correspondence to Dr. Xiao-Yan Wang, Department of Cariology and Endodontology, Peking University School and Hospital of Stomatology, 22 Zhongguancun Avenue South, Haidian District, Beijing 100081, P. R. China

Fax: +86-010-62173402

E-mail: wangxiaoyan@pkuss.bjmu.edu.cn

J-STAGE Advance Publication: November 17, 2017

doi.org/10.2334/josnusd.16-0636

DN/JST.JSTAGE/josnusd/16-0636 was more effective in lightening teeth and reducing chromacity in Chinese patients. The correlation between tooth color change and outcome satisfaction was generally insignificant and weak.

Keywords: dental bleaching; Chinese patient; spectrophotometer; outcome satisfaction.

\section{Introduction}

Intrinsic and extrinsic tooth discoloration are commonly encountered in routine dental practice. Teeth bleaching is a safe and conservative method of treating discolored teeth (1). Home and in-office teeth bleaching are the two main professionally prescribed techniques. Patients perform home bleaching (HB) at home, usually with a customized tray filled with bleaching gel; it is typically administered after dinner or overnight for a week or two. The bleaching gel employed is typically carbamide peroxide (CP) with a concentration of $5-35 \%(2-5)$. In-office bleaching is clinically performed by dentists and does not involve wearing of bleaching trays. Treatment time is much shorter than HB, and in-office bleaching is completed in a single clinical visit. In-office bleaching uses a high-concentration hydrogen peroxide (HP) gel with a concentration ranging between $15 \%$ and $40 \%$, which is often illuminated with a light source $(6,7)$. Both aforementioned gel types break down into water, oxygen, and perhydroxyl anion. While HP gels begin this breakdown directly, CP gels must first be broken down into urea and HP. Despite the higher concentration of HP present in in-office bleaching systems, their effi- 
cacy remains equivocal to the lower-concentration $\mathrm{HB}$ systems (3,7-9).

Bleaching efficacy is primarily determined by the degree of tooth color change and is often measured against a standardized dental shade guide arranged according to values i.e., number of shades lighter $(2,6,10)$. This approach of assessing bleaching efficacy is somewhat subjective and not quantifiable. The three-parameter Commission Internationale de l'Eclairage (CIE) LAB system is a globally used standard that assigns numerical values to define color. The $\mathrm{L}^{*}$ coordinate is related to lightness with the $\mathrm{a}^{*}$ coordinate to red (more positive) and green (more negative), whereas the $b^{*}$ coordinate is related to yellow (more positive) and blue (more negative) (11). Spectrophotometers have adopted this measurement system and allow for objective, precise, and quantifiable measurement of tooth color (12).

The world's major racial groups can be broadly classified into Caucasoids, Mongoloids (Asian), Negroids, and Australoids (Australian aborigines). Most clinical trials on dental bleaching have been conducted on Caucasian populations (9,13-19). However, racial differences in tooth anatomy, dimensions, and translucency may influence the outcomes of dental bleaching (20-23). Available literature on comparison of the degree of tooth color change associated with different bleaching techniques in Asian populations is scarce as are studies evaluating outcome satisfaction and preference of dental bleaching techniques among Asian patients (24). With increased popularity and demand for tooth bleaching by Asian patients, bleaching efficacy and patients' outcome satisfaction warrants additional investigation.

The objectives of this study conducted in Chinese patients were to i) compare the color change between in-office and $\mathrm{HB}$ by using objective spectrophotometry and subjective patient assessment (outcome satisfaction); ii) compare the differences in color change associated with bleaching between incisors and canines; iii) compare short- and long-term color stability of in-office and HB; and iv) determine correlation of color changes with patients' outcome satisfaction.

\section{Materials and Methods}

Before initiation, study approval was obtained from the Dental Institute Research Ethics Committee at Peking University (PKUSSIRB-2012035). Asian subjects of Chinese descent were recruited from patients seeking bleaching treatment at the Peking University Hospital of Stomatology. Patients with vital and periodontally sound teeth alone were selected. Those with carious, extensively restored, root canal-treated, periodontally compromised, traumatized, and/or grossly malformed teeth were excluded. In addition, patients with tetracycline staining/fluorosis and those who were pregnant or suffering from systemic diseases were excluded. All patients who qualified were provided details of the study, a patient information sheet, and were required to provide signed informed consent before study enrolment. All patients were provided the option to withdraw from the study at any time.

A total of 40 patients (10 men and 30 women) with a mean age of 27 years (range: 21-39 years) were recruited in the study. Oral hygiene instructions were given, and any extrinsic stains were removed by polishing with pumice. Subsequently, subjects were randomly divided into two groups of 20 each. Patients in the first group were treated with in-office bleaching $(\mathrm{OB})$ with Opalescence Boost (38\% HP; Ultradent, South Jordan, UT, USA), while those in the second group received HB with Opalescence PF (10\% CP; Ultradent). In the OB group, soft tissues were isolated/protected with a light-cure resin (OpalDam; Ultradent) that was polymerized using an LED light with an intensity of $500 \mathrm{~mW} / \mathrm{cm}^{2}$ (Ivoclar Vivadent, Schaan, Liechtenstein). The bleaching gel was then applied in 1-mm thickness onto buccal surfaces of the teeth according to manufacturer's instructions. In-office bleaching consisted of three 15-min bleaching cycles involving placement and removal of fresh gel. The total bleaching time was $45 \mathrm{~min}$.

Customized bleaching trays were fabricated for patients from the HB group. Patients were taught to fill half of the buccal surfaces of the bleaching trays with bleaching gel and to wear the trays after brushing their teeth. They were instructed to bleach their teeth every night for $8 \mathrm{~h}$ for 12 consecutive nights. Patients were asked to stop HB for a day if case sensitivity occurred and to continue after tooth sensitivity subsides. If patients were unable to continue HB because of severe sensitivity, they were provided the option to stop $\mathrm{HB}$ and withdraw from the study.

For both OB and HB groups, $\mathrm{L}^{*}, \mathrm{a}^{*}$, and $\mathrm{b}^{*}$ values of maxillary central incisors and canines were recorded using the Crystaleye Spectrophotometer (Olympus, Tokyo, Japan) before bleaching (baseline), immediately after the first treatment (imm), 1 week $(1 \mathrm{w})$, and 3 months $(3 \mathrm{~m})$ after bleaching treatment. The total color change at these different time points was computed using the formula: $\Delta \mathrm{E}^{*}=\left[\left(\Delta \mathrm{L}^{*}\right)^{2}+\left(\Delta \mathrm{a}^{*}\right)^{2}+\left(\Delta \mathrm{b}^{*}\right)^{2}\right]^{1 / 2}$.

Patients were asked to rate their tooth sensitivity and treatment outcome satisfaction immediately, 1 week, and 3 months after in-office and HB by means of a four-point Likert ordinal scale (no, mild, moderate, and 
Table 1 Baseline color of incisors and canines in both OB and HB groups

\begin{tabular}{cccccccc}
\hline & \multicolumn{3}{c}{ Incisor } & & \multicolumn{3}{c}{ Canine } \\
\cline { 2 - 3 } \cline { 6 - 7 } & $\mathrm{L}^{*}$ & $\mathrm{a}^{*}$ & $\mathrm{~b}^{*}$ & & $\mathrm{~L}^{*}$ & $\mathrm{a}^{*}$ & $\mathrm{~b}^{*}$ \\
\hline OB & $72.96 \pm 2.55$ & $1.34 \pm 0.73$ & $17.70 \pm 2.70$ & & $69.64 \pm 2.32$ & $3.50 \pm 1.26$ & $21.73 \pm 3.29$ \\
HB & $73.34 \pm 2.53$ & $1.18 \pm 0.90$ & $17.76 \pm 3.23$ & & $69.14 \pm 2.35$ & $4.11 \pm 0.89$ & $24.31 \pm 2.14$ \\
\hline
\end{tabular}

(mean \pm standard deviation)

No statistically significant differences (results of independent $t$-test, 2-tailed). OB: in-office bleaching; HB: home bleaching.

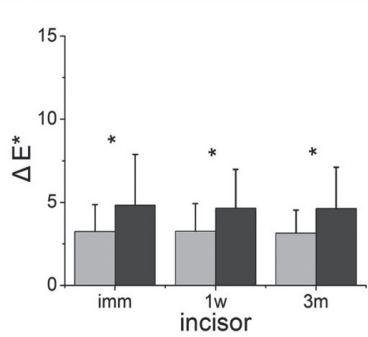

(A)

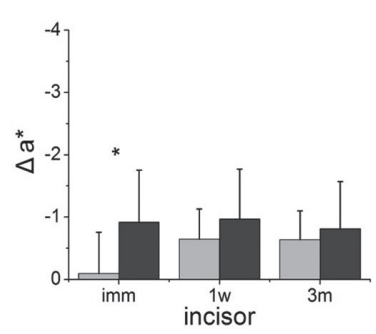

(C)
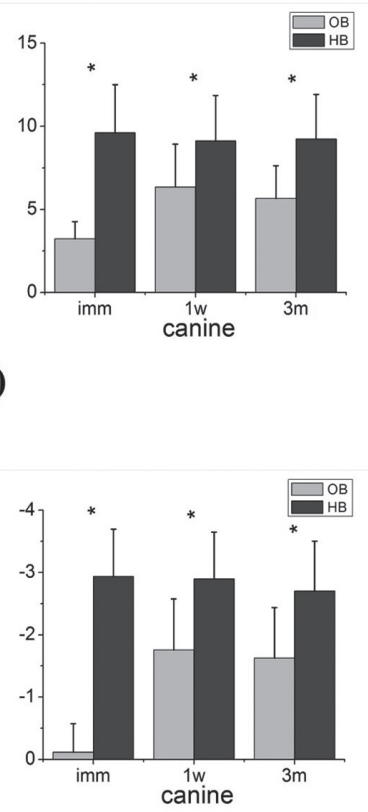

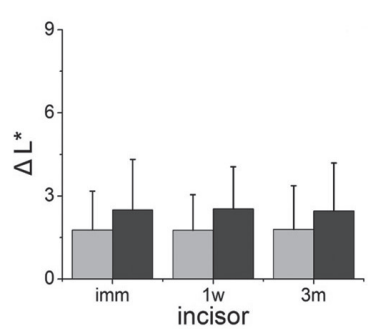

(B)
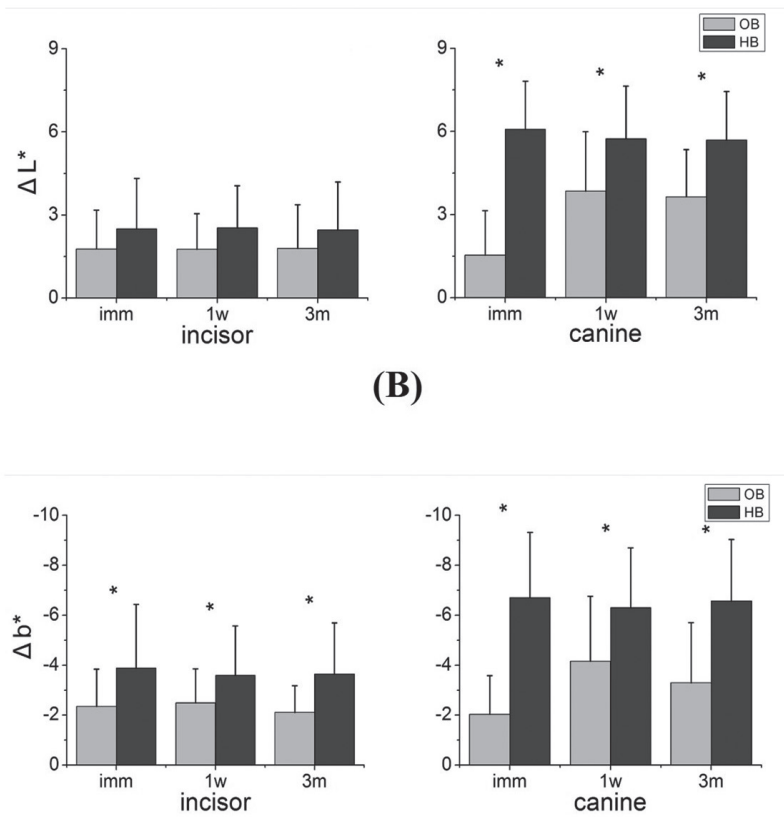

(D)

Fig. 1 Comparison of $\Delta \mathrm{E}^{*}(\mathrm{~A}), \Delta \mathrm{L}^{*}, \Delta \mathrm{a}^{*}, \Delta \mathrm{b}^{*}$ values for incisors and canines at the three study time points. (B) Positive $\Delta \mathrm{L}^{*}$ values were observed, indicating increased value or "brightness" after bleaching. (C) Negative $\Delta \mathrm{a}^{*}$, signifying decreased chromacity, was noted in both bleaching groups. (D) Negative $\Delta \mathrm{b}^{*}$, signifying decreased chromacity (less yellow), was noted in both bleaching groups. *Indicates statistically significant differences $(P<0.05$, results of independent $t$-test, 2 -taile).

severe tooth sensitivity) and a 010-point visual analog scale (VAS), respectively. VAS scores were classified as follows: highly satisfied (HS), 8-10 points; satisfied (S), 5-7 points; and not satisfied (NS), $<5$ points. Statistical analysis was performed using SPSS 20 (IBM, Armonk, NY, USA). Spectrophotometric data were analyzed using independent $t$-tests, while correlation between color change and patients' outcome satisfaction was determined using Pearson correlation $(P<0.05)$.

\section{Results}

No statistically significant differences were noted in the mean baseline color of incisors and canines between $\mathrm{OB}$ and HB groups (Table 1). In general, color changes noted in canines were greater than those in incisors. In addition, $\Delta \mathrm{E}^{*}$ values of $\mathrm{HB}$ were significantly higher than those of $\mathrm{OB}$ at all three time points for both incisors and canines (Fig. 1A). The $\Delta \mathrm{E}^{*}$ values remained stable at 1 week and
3 months, with significant differences between $\mathrm{HB}$ and OB. Moreover, similar trends were observed for $\Delta \mathrm{L}^{*}$, $\Delta \mathrm{a}^{*}$, and $\Delta \mathrm{b}^{*}$ parameters. Statistically significant differences observed in $\Delta \mathrm{E}^{*}, \Delta \mathrm{L}^{*}, \Delta \mathrm{a}^{*}$, and $\Delta \mathrm{b}^{*}$ parameters are reflected in Fig. 1A-D.

The distribution of patients' tooth sensitivity and outcome satisfaction is shown in Tables 2 and 3. Patients from the $\mathrm{HB}$ group reported less tooth sensitivity compared with those from the OB group. Three months after starting bleaching, three HB group patients reported mild sensitivity, and one $\mathrm{OB}$ group patient reported severe tooth sensitivity. Mean VAS scores were $>7$ for $\mathrm{OB}$ and 8 for HB. A greater proportion of HB group patients were satisfied compared with $\mathrm{OB}$ group patients.

No significant correlation was identified between $\Delta \mathrm{E}^{*}$ and patients' outcome satisfaction for incisors. In contrast, for canines, the correlation between color change and patient satisfaction was significant and 
Table 2 Percentage of tooth sensitivity assessed using a four-point Likert ordinal scale

\begin{tabular}{lcccccccc}
\hline & \multicolumn{2}{c}{$1=$ No sensitivity } & \multicolumn{2}{c}{$2=$ Mild } & \multicolumn{2}{c}{$3=$ Moderate } & \multicolumn{2}{c}{$4=$ Severe } \\
\cline { 2 - 8 } & OB & HB & OB & HB & OB & HB & OB & HB \\
\hline imm & $50 \%$ & $80 \%$ & $35 \%$ & $15 \%$ & $15 \%$ & $5 \%$ & 0 & 0 \\
1w & $90 \%$ & $100 \%$ & 0 & 0 & $10 \%$ & 0 & 0 & 0 \\
$3 \mathrm{~m}$ & $95 \%$ & $85 \%$ & 0 & $15 \%$ & 0 & 0 & $5 \%$ & 0 \\
\hline \multicolumn{2}{l}{ imm: immediately after teeth bleaching; 1w: 1 week after teeth bleaching; 3m: 3 months after teeth bleaching. }
\end{tabular}

Table 3 Proportion of patients who were highly satisfied, satisfied, and not satisfied with their bleaching outcomes and VAS scores

\begin{tabular}{lccccccccc}
\hline & \multicolumn{2}{c}{ HS } & \multicolumn{2}{c}{$\mathrm{S}$} & \multicolumn{2}{c}{ NS } & \multicolumn{2}{c}{ Mean VAS score } \\
\cline { 2 - 10 } & OB & HB & OB & HB & OB & HB & OB & HB \\
\hline imm & $50 \%$ & $85 \%$ & $50 \%$ & $15 \%$ & 0 & 0 & $7.3 \pm 1.7$ & $8.6 \pm 1.4$ \\
$1 \mathrm{w}$ & $50 \%$ & $85 \%$ & $40 \%$ & $15 \%$ & $10 \%$ & 0 & $7.3 \pm 2.2$ & $8.6 \pm 1.2$ \\
$3 \mathrm{~m}$ & $45 \%$ & $85 \%$ & $40 \%$ & $15 \%$ & $15 \%$ & 0 & $7.0 \pm 2.4$ & $8.3 \pm 1.9$ \\
\hline
\end{tabular}

(mean \pm standard deviation)

HS: highly satisfied; S: satisfied; NS: not satisfied.

Table 4 Correlation between color change $\left(\Delta \mathrm{E}^{*}\right)$ and patients' outcome satisfaction (VAS scores)

\begin{tabular}{|c|c|c|c|}
\hline & & OB & HB \\
\hline & & $\mathrm{r}$ & $\mathrm{r}$ \\
\hline \multirow{3}{*}{ Incisor } & $\mathrm{imm}$ & -0.157 & 0.075 \\
\hline & $1 \mathrm{w}$ & 0.162 & 0.088 \\
\hline & $3 \mathrm{~m}$ & 0.083 & 0.266 \\
\hline \multirow{3}{*}{ Canine } & $\mathrm{imm}$ & 0.138 & $0.628 *$ \\
\hline & $1 \mathrm{w}$ & 0.191 & $0.489 *$ \\
\hline & $3 \mathrm{~m}$ & 0.395 & 0.386 \\
\hline
\end{tabular}

relatively strong immediately after bleaching, which continued to remain significant 1 week after bleaching in the HB group (Table 4). The correlation was insignificant and weak for incisors in both OB and HB groups as well as for canines in the OB group.

\section{Discussion}

In the present study, maxillary central incisors and canines were selected because maxillary teeth are more visually prominent and "yellowish" than mandibular teeth (25). In addition, the color difference between central incisors and canines is generally larger than that between central and lateral incisors (26). The perceptible threshold of color change by the human eye was reported to range from $\Delta \mathrm{E}^{*}$ of 1 to 2.6 under clinical conditions (27). As the $\Delta \mathrm{E}^{*}$ associated with both $\mathrm{OB}$ and $\mathrm{HB}$ was $>3$ for both teeth, color changes were clinically distinguishable. In this study, color changes noted in canines after bleaching were greater than those in incisors. The $\Delta \mathrm{E}^{*}$ values obtained corroborated those reported by
Costa et al. and Nathoo et al., who reported a mean $\Delta \mathrm{E}^{*}$ of approximately 5.2 and 4.05 for products containing $10 \% \mathrm{CP}$ at 1 week after bleaching $(2,9)$. Tooth color after bleaching remained relatively stable and did not change much at 3 months. Tsubara et al. reported a $\Delta \mathrm{E}^{*}$ of 13.74 for canine teeth at 3 months after $\mathrm{HB}$ with $10 \% \mathrm{CP}$ gel by using a portable chroma meter; this $\Delta \mathrm{E}^{*}$ value remained stable even 2 years after treatment (28). Collectively, the results suggest that the mid- to long-term color stability of $\mathrm{HB}$, which has implications for restorative treatment planning, is predictable and reliable.

In-office bleaching is a practical alternative therapy for patients who are unable to comply with $\mathrm{HB}$ regimes, do not like wearing bleaching trays, or wish to instantaneously have whiter teeth. Salem et al. evaluated the color change of maxillary central incisors after a single session of in-office vital teeth bleaching with $35 \% \mathrm{HP}$ and reported a $\Delta \mathrm{E}^{*}$ value of 3.56 (29); this value was comparable to that obtained in the present study. As with the HB group, color changes observed in canines were greater than those noted in incisors. In fact, Wetter et al. reported greater color changes in canines for both $\mathrm{HB}$ and $\mathrm{OB}$ in a bleaching study involving Caucasian patients (30). This observation may be partly attributed to the greater color saturation of canines than incisors. In the present study, the $\Delta \mathrm{E}^{*}$ values obtained with $\mathrm{OB}$ were significantly lower than those with HB for both incisors and canines. Hence, a single session of in-office bleaching is not as effective as a course of HB for "whitening" Chinese teeth. This finding is consistent with that documented by Zeknois et al., who reported that HB produced significantly lighter teeth than in-office bleaching during all active treatment periods and follow-up visits (10). However, most other 
bleaching studies conducted in Caucasian populations have reported both $\mathrm{HB}$ and $\mathrm{OB}$ to be equally effective (9,13-19). The disparity in findings may be attributed to differences in bleaching agent concentrations, total treatment time, and racial variance of human teeth (20-23). Bleaching studies that compared different Asian ethnic groups (e.g., Chinese, Koreans, Japanese, and Malays) will be more relevant as dissimilarities arising from genetic alternations and environmental influences are anticipated. A two-fold increase in $\Delta \mathrm{E}^{*}(3.23-6.35)$ was observed in canines immediately after the first treatment and 1 week after $\mathrm{OB}$; this phenomenon may be explained by remnant perhydroxyl radicals in the tooth structure arising from the high concentrations of bleaching agent used $(31,32)$.

HB was associated with a lower frequency of tooth sensitivity compared with OB in Chinese patients. Similar findings have been reported in previous Caucasian studies and can be ascribed to the higher peroxide concentrations $(15,17)$. For most patients, tooth sensitivity, when present, was predominantly mild and transient. Nevertheless, some patients reported tooth sensitivity even at the 3-month study time point, but these were essentially associated with cervical defects and root surface exposure and not the bleaching treatment. Since patients may confuse transient tooth sensitivity from bleaching with dentin hypersensitivity from cervical defects/root exposure, it is prudent to carefully examine teeth for the latter and to address such concerns before bleaching. Leonard et al. reported that patients with sensitivity after oral prophylaxis are more likely to encounter sensitivity and other side effects during bleaching. In fact, it was advised that bleaching procedures should be delayed for 2 weeks after prophylaxis treatment (33).

In this study, a VAS was used to provide a quantitative scale of satisfaction $(3,34)$. Based on VAS scores recorded at various time intervals, a majority of patients were more satisfied with the results of $\mathrm{HB}$ than of OB. Similar outcomes have been reported in previous Caucasian studies, and most patients preferred and would recommend $\mathrm{HB}$ over OB $(10,14)$. Matis et al. examined patients' outcome satisfaction with HB by means of a questionnaire for tetracycline-stained teeth treated using different concentrations of $\mathrm{CP}(35)$. Up to $88 \%$ of subjects were "pleased" with the outcome, and $41 \%$ remained "pleased" with how their teeth looked after 5 years.

Previous studies have indicated that tooth bleaching works by increasing $L^{*}$ values and decreasing $b^{*}$ values $(9,28,36)$. Both OB and HB increased "lightness" $\left(\Delta \mathrm{L}^{*}\right)$ and decreased "yellowness" $\left(\Delta \mathrm{b}^{*}\right)$ and "redness" $\left(\Delta \mathrm{a}^{*}\right)$ at all three study time points. Therefore, teeth were observed to be whiter and less color intense by patients after both bleaching treatments. The correlation between tooth color change and patient satisfaction has not been widely investigated. For both $\mathrm{OB}$ and $\mathrm{HB}$, no significant correlation was noted between $\Delta \mathrm{E}^{*}$ and patients' outcome satisfaction for incisors at all three study time points. Although no significant correlation was observed for canines in the OB group, a significant correlation was noted in the HB group immediately and 1 week after bleaching. Taken together, the findings of this study establish that objective color changes cannot be used as a parameter to predict patients' bleaching outcome satisfaction.

Within the limitations of this in vivo study, the following conclusions can be made: a) color changes, tooth sensitivity, and outcome satisfaction of tooth bleaching in Chinese patients were similar to those reported in Caucasian patients. Unlike most Caucasian studies where the effects of $\mathrm{HB}$ and $\mathrm{OB}$ were reported to be comparable, HB was found to be more effective than $\mathrm{OB}$ in Chinese patients; $b$ ) color changes noted in canines were generally greater than those in incisors; c) Chinese patients were satisfied with both bleaching treatments, but outcome satisfaction scores for HB were higher; d) the correlation between tooth color change and outcome satisfaction was generally insignificant and weak.

\section{Acknowledgments}

The authors would like to thank Ultradent for sponsorship of the materials used in the study.

\section{Conflict of interest}

The authors do not have any financial interest in the companies whose materials are included in this article.

\section{References}

1. Burrows S (2009) A review of the safety of tooth bleaching. Dent Update 36, 604-614.

2. Nathoo S, Santana ER 3rd, Zhang YP, Lin N, Collins M, Klimpel K et al. (2001) Comparative seven-day clinical evaluation of two tooth whitening products. Compend Contin Educ Dent 22, 599-604, 606.

3. Krug AY, Green C (2008) Changes in patient evaluation of completed orthodontic esthetics after dental bleaching. J Esthet Restor Dent 20, 313-321.

4. da Costa JB, McPharlin R, Hilton T, Ferracane JI, Wang M (2012) Comparison of two at-home whitening products of similar peroxide concentration and different delivery methods. Oper Dent 37, 333-339.

5. Soares DG, Basso FG, Hebling J, de Souza Costa CA (2014) Concentrations of and application protocols for hydrogen peroxide bleaching gels: effects on pulp cell viability and 
whitening efficacy. J Dent 42, 185-198.

6. Alomari Q, El Daraa E (2010) A randomized clinical trial of in-office dental bleaching with or without light activation. J Contemp Dent Pract 11, E017-24.

7. Nutter BJ, Sharif MO, Smith AB, Brunton PA (2013) A clinical study comparing the efficacy of light activated in-surgery whitening versus in-surgery whitening without light activation. J Dent 41, Suppl 5, e3-7.

8. Knösel M, Attin R, Becker K, Attin T (2008) A randomized CIE L*a*b* evaluation of external bleaching therapy effects on fluorotic enamel stains. Quintessence Int 39, 391-399.

9. da Costa JB, McPharlin R, Paravina RD, Ferracane JL (2010) Comparison of at-home and in-office tooth whitening using a novel shade guide. Oper Dent 35, 381-388.

10. Zekonis R, Matis BA, Cochran MA, Al Shetri SE, Eckert GJ, Carlson TJ (2003) Clinical evaluation of in-office and at-home bleaching treatments. Oper Dent 28, 114-121.

11. O’Brien WJ, Groh CL, Boenke KM (1990) A new, small-color-difference equation for dental shades. J Dent Res 69, 1762-1764.

12. Goodson JM, Tavares M, Sweeney M, Stultz J, Newman M, Smith V et al. (2005) Tooth whitening: tooth color changes following treatment by peroxide and light. J Clin Dent 16 , 78-82.

13. Bizhang M, Chun YH, Damerau K, Singh P, Raab WH, Zimmer S (2009) Comparative clinical study of the effectiveness of three different bleaching methods. Oper Dent 34, 635-641.

14. Giachetti L, Bertini F, Bambi C, Nieri M, Scaminaci Russo D (2010) A randomized clinical trial comparing at-home and in-office tooth whitening techniques: a nine-month followup. J Am Dent Assoc 141, 1357-1364.

15. Dawson PF, Sharif MO, Smith AB, Brunton PA (2011) A clinical study comparing the efficacy and sensitivity of home vs combined whitening. Oper Dent 36, 460-466.

16. Almeida LC, Riehl H, Santos PH, Sundfeld ML, Briso AL (2012) Clinical evaluation of the effectiveness of different bleaching therapies in vital teeth. Int J Periodontics Restorative Dent 32, 303-309.

17. Basting RT, Amaral FL, França FM, Flório FM (2012) Clinical comparative study of the effectiveness of and tooth sensitivity to $10 \%$ and $20 \%$ carbamide peroxide home-use and $35 \%$ and $38 \%$ hydrogen peroxide in-office bleaching materials containing desensitizing agents. Oper Dent 37, 464-473.

18. Mondelli RF, Azevedo JF, Francisconi AC, Almeida CM, Ishikiriama SK (2012) Comparative clinical study of the effectiveness of different dental bleaching methods--two year follow-up. J Appl Oral Sci 20, 435-443.

19. Tay LY, Kose C, Herrera DR, Reis A, Loguercio AD (2012) Long-term efficacy of in-office and at-home bleaching: a 2-year double-blind randomized clinical trial. Am J Dent 25, 199-204.

20. Yaacob H, Nambiar P, Naidu MD (1996) Racial characteris- tics of human teeth with special emphasis on the Mongoloid dentition. Malays J Pathol 18, 1-7.

21. Purton DG, Ng BP, Chandler NP, Monteith BD (2004) The bitewing radiograph as an assessment tool in fixed prosthodontics. J Oral Rehabil 31, 562-567.

22. Brook AH, Griffin RC, Townsend G, Levisianos Y, Russell J, Smith RN (2009) Variability and patterning in permanent tooth size of four human ethnic groups. Arch Oral Biol 54, Suppl 1, S79-85.

23. Bayindir F, Gozalo-Diaz D, Kim-Pusateri S, Wee AG (2012) Incisal translucency of vital natural unrestored teeth: a clinical study. J Esthet Restor Dent 24, 335-343.

24. Zhao K, Zong L, Zhang Q, Att W (2013) Clinical comparison between two bleaching techniques: a 180-day follow-up study. Quintessence Int 44, 601-607.

25. Goodkind RJ, Schwabacher WB (1987) Use of a fiber-optic colorimeter for in vivo color measurements of 2830 anterior teeth. J Prosthet Dent 58, 535-542.

26. Dozic A, Kleverlaan CJ, Aartman IH, Feilzer AJ (2005) Relations in color among maxillary incisors and canines. Dent Mater 21, 187-191.

27. Douglas RD, Steinhauer TJ, Wee AG (2007) Intraoral determination of the tolerance of dentists for perceptibility and acceptability of shade mismatch. J Prosthet Dent 97, 200-208.

28. Tsubura S (2010) Clinical evaluation of three months' nightguard vital bleaching on tetracycline-stained teeth using Polanight $10 \%$ carbamide gel: 2-year follow-up study. Odontology 98, 134-138.

29. Salem YM, Osman YI (2011) The effect of in-office vital bleaching and patients' perception of the shade change. SADJ 66, 70-76.

30. Wetter NU, Branco EP, Deana AM, Pelino JE (2009) Color differences of canines and incisors in a comparative longterm clinical trial of three bleaching systems. Lasers Med Sci 24, 941-947.

31. Rotstein I (1993) Role of catalase in the elimination of residual hydrogen peroxide following tooth bleaching. J Endod 19, 567-569.

32. Kaya AD, Turkun M, Arici M (2008) Reversal of compromised bonding in bleached enamel using antioxidant gel. Oper Dent 33, 441-447.

33. Leonard RH Jr (1998) Efficacy, longevity, side effects, and patient perceptions of nightguard vital bleaching. Compend Contin Educ Dent 19, 766-770, 772, 774.

34. Miller MD, Ferris DG (1993) Measurement of subjective phenomena in primary care research: the Visual Analogue Scale. Fam Pract Res J 13, 15-24.

35. Matis BA, Wang Y, Eckert GJ, Cochran MA, Jiang T (2006) Extended bleaching of tetracycline-stained teeth: a 5-year study. Oper Dent 31, 643-651.

36. Ishikawa-Nagai S, Yoshida A, Da Silva JD, Miller L (2010) Spectrophotometric analysis of tooth color reproduction on anterior all-ceramic crowns: Part 1: analysis and interpretation of tooth color. J Esthet Restor Dent 22, 42-52. 